\title{
EL ROL DEL PRINCIPIO DE COMPETENCE- COMPETENCE EN LA PREVENCIÓN DE TÁCTICAS DILATORIAS EN EL ARBITRAJE COMERCIAL INTERNACIONAL
}

\author{
THE ROLE OF THE "COMPÉTENCE - \\ COMPÉTENCE” PRINCIPLE ON THE \\ PREVENTION OF DILATORY TACTICS IN THE \\ INTERNATIONAL COMMERCIAL ARBITRATION
}

\author{
Shirley Llain Arenilla**
}

PARA CITAR este ARTículo / To CITE THIS ARTICLE Llain Arenilla, S., El rol del principio de "competence-competence" en la prevención de tácticas dilatorias en el arbitraje comercial internacional, 24 International Law, Revista Colombiana de Derecho Internacional, PÁGPÁG (2014).

doi:10.11144/Javeriana.IL14-24.rpcc

* Artículo de investigación dentro del marco del grupo de investigaciones en Derecho y Ciencia Política de la Universidad del Norte.

** Abogada, Universidad del Norte. Master of Law (LL.M.) in International Law, Stetson University College of Law. Especialista en Derecho Comercial, Universidad Externado de Colombia, Especialista en Derecho Comercial, Universidad del Norte, Diplomado en Arbitraje Comercial Internacional, Cámara de Comercio de Bogotá, XXXVIII Curso de Derecho Internacional, oea y Comité Jurídico Interamericano. Actualmente es docente investigadora de la División de Ciencias Jurídicas de la Universidad del Norte. Barranquilla, Colombia. Correo electrónico: sllain@uninorte.edu.co 


\section{RESUMEN}

La utilización de maniobras dilatorias, por partes recalcitrantes, se ha convertido en una realidad del arbitraje comercial internacional. Con el presente artículo, mediante un método de investigación cualitativa, fundamentado en la revisión crítica de literatura y casuística, nos proponemos mostrar que esas tácticas pueden combatirse mediante la aplicación del principio de competence-competence, desde sus dos efectos: positivo y negativo, establecidos tanto en la doctrina arbitral como en la ley modelo de CNUDMI, así como en diversas convenciones y reglas sobre arbitraje comercial internacional. Para tales efectos, en primera instancia, se estudiará el principio de competence-competence, debido a que supone un mínimo de supervisión por parte de las cortes judiciales, y le otorga facultades al tribunal arbitral para guiar el proceso arbitral hasta su etapa decisoria sin mayores demoras; en segunda instancia, se presentarán distintos tipos de tácticas dilatorias que las partes emplean en los procesos arbitrales para impugnar sin justa causa la jurisdicción del tribunal arbitral, y analizar los mecanismos o herramientas que, con base en las facultades de los árbitros, derivadas del principio de competence-competence pueden ser utilizados para contrarrestarlas.

Palabras clave autora: Competence-competence, tácticas dilatorias, arbitraje comercial internacional, pacto arbitral, impugnación de jurisdicción, mala fe. Palabras clave descriptores: Competencia, arbitramento comercial, mala fe, jurisdicción, recursos (Derecho). 


\section{Abstract}

The use of dilatory tactics by recalcitrant parties has become a reality of the international commercial arbitration. The purpose of this article, using a qualitative method of research based in the critical review of literature and case study, is to show that such tactics can be prevent through the application of the Competence - Competence Principle, from the point of view of its two effects: positive and negative, settled in the arbitral doctrine and several conventions and rules on international commercial arbitration. Thus, first, we will study the Competence - Competence Principle because it supposes a minimum of supervision by the judicial courts, and it gives faculties to the arbitral tribunal to guide the arbitral process until its decision stage without further delay; second, we will show types of dilatory tactics employed by the parties in the arbitral procedures to impugn without cause the lack of jurisdiction of the arbitral tribunal, and finally we will analyze the mechanisms or tools based in the faculties of the arbitrators that may be used to counteract them.

Keywords author: "Competence-Competence", dilatorytactics, international arbitration, arbitral agreement, challenge of jurisdiction, bad faith

Keywords plus: Competition, commercial arbitration, bad faith (law), jurisdiction, remedies (law).

\section{SUMARIO}

INTRODUCCIÓN.-I. EL PRINCIPIO DE COMPETENCE-COMPETENCE.-II. RELACIÓN DEL PRINCIPIO DE COMPETENCE-COMPETENCE CON TÁCTICAS DILATORIAS EMPLEADAS POR LaS PARTES EN El PRoceso ARBitral.- A. Efecto positivo del principio de competence-competence y su relación con las tácticas dilatorias.- B. Efecto negativo del principio de competence-competence y su relación con las tácticas dilatorias. C. Tácticas dilatorias relacionadas con la competencia del tribunal arbitral.- III. Conclusión.- Bibliografía. 


\section{INTRODUCCIÓN}

El arbitraje comercial internacional es hoy en día uno de los mecanismos de solución de conflicto más utilizados en materia de negocios internacionales. Sin duda, cualidades como la idoneidad de los árbitros, la rapidez y eficiencia en la resolución del conflicto, los mecanismos para el reconocimiento y ejecución de los laudos arbitrales y la confidencialidad, entre otras, atraen a este foro a un sinnúmero de empresarios que, por efectos de la globalización de la economía, buscan soluciones ajustados a la velocidad con que los negocios se mueven actualmente. Sin embargo, estas ventajas pueden verse menguadas cuando en los procesos arbitrales se encuentran partes dispuestas a utilizar una variedad de trucos y tácticas para dilatar el inicio o avance del proceso, e interponen recursos infundados, bien sea ante los mismos tribunales arbitrales o ante las cortes nacionales de las jurisdicciones competentes.

Este tipo de conductas se convierte no solo en una barrera para el proceso arbitral sino en una situación que puede llegar a desestimular el uso del arbitraje internacional, debido a la pérdida de agilidad y eficiencia que lo han caracterizado.

Nace entonces, para todos aquellos que cumplen un papel en el arbitraje comercial internacional — partes, árbitros y jueces-, la necesidad de analizar de forma detallada cuáles son las actitudes procesales que las partes adoptan para sabotear un proceso arbitral, y cuáles son las herramientas que las legislaciones arbitrales, convenciones internacionales, reglas de instituciones de arbitraje internacional y la propia doctrina arbitral brindan para contrarrestarlas.

En este marco, el principio de competence-competence desempeña un rol esencial, pues se presenta como la principal herramienta que los mencionados actores del arbitraje comercial internacional tienen para evitar el uso de tácticas dilatorias. Estas tácticas son aplicadas por partes recalcitrantes o de mala fe para atacar el acuerdo arbitral pactado, debido a que supone un mínimo de supervisión de las cortes judiciales, y le otorga facultades 
al tribunal arbitral para guiar el proceso arbitral hasta su etapa decisoria sin mayores demoras.

De esta manera, los árbitros pueden combatir tácticas dilatorias tendientes a cuestionar la validez del acuerdo arbitral y, por ende, la competencia del tribunal arbitral, de manera que pueden decidir sobre su propia competencia y continuar con el proceso arbitral, aunque la parte que pretende retardar el proceso acuda a instancias jurisdiccionales.

Al respecto, la Ley Modelo de Arbitraje de la CNUDMI (Comisión de las Naciones Unidas para el Derecho Mercantil) y distintos reglamentos de instituciones de arbitraje internacional prevén mecanismos en desarrollo del principio de competencecompetence, con la mencionada finalidad, entre los que encontramos: la imposición de plazos para impugnar la competencia del tribunal arbitral; la inapelabilidad de la decisión del tribunal arbitral; y la inclusión del reglamento de la institución arbitral escogida por las partes en el contrato, de tal forma que se entienda que, al aceptar el arbitraje bajo ese reglamento, las partes convienen en no acudir a cortes estatales o utilizar recursos judiciales para discutir la jurisdicción del tribunal arbitral.

Existe entonces una gran variedad de tácticas dilatorias, como expondremos más adelante, pero también una gran variedad de recursos y facultades dadas a los árbitros por medio del principio de competence-competence, que les permiten favorecer el avance del proceso arbitral. Tal como lo señala L. Yves Fortier, "the broad discretion granted to the arbitrator to determine the conduct of proceedings arms him, in effect, with a full quiver of arrows to be deployed in the fight against dilatory tactics".

1 L. Y. Fortier, The Minimum Requirements of Due Process in Taking Measures against Dilatory Tactics: Arbitral Discretion in International Commercial Arbitration. A Few Plain Rules and a Few Strong Instincts en Improving the Efficiency of Arbitration Agreements and Awards: 40 Years of Application of the New York Convention, 396 (A. J. van Den Berg, Ed., Kluwer Law International, The Hague, 1999). 


\section{EL PRINCIPIO DE COMPETENCE-COMPETENCE}

El principio de competence-competence es uno de los pilares fundamentales del arbitraje comercial internacional y, al mismo tiempo, el más controversial en el ámbito legislativo y doctrinario, en la medida en que discute los alcances de la jurisdicción del tribunal arbitral y quién debe decidir su competencia. En virtud de este principio "los árbitros son los jueces provisionales de su propia competencia para adelantar el proceso arbitral y para proferir el laudo que pone fin a la controversia entre las partes" 2 . Es decir, los árbitros tienen la facultad para fijar en forma preliminar quién posee la autoridad para fallar sobre el fondo de una determinada disputa ${ }^{3}$. En este sentido, la decisión que tome un tribunal arbitral estará sujeta a la posterior revisión de las cortes nacionales ${ }^{4}$.

El principio de competence- competence va de la mano del denominado principio de separabilidad o de autonomía del convenio arbitral, según el cual, la cláusula arbitral es independiente del contrato principal que la contiene y, por ende, los árbitros poseen plena facultad para resolver controversias relativas a la existencia y validez del contrato principal. De esta forma, si el contrato principal está afectado de nulidad, la cláusula arbitral no corre igual suerte ${ }^{5}$. La diferencia entre estos dos principios reside entonces en que, según el principio de separabilidad, los árbitros tienen la facultad de resolver controversias relativas a la existencia y validez del contrato principal, mientras que de acuerdo con el principio de competence-competence, los árbitros

2 S. Talero Rueda, Arbitraje comercial internacional. Instituciones básicas y derecho aplicable, 147 (Ediciones Uniandes, Temis, Bogotá D.C., 2008).

3 Según la doctrina arbitral, el principio de competence-competence es una regla de prioridad cronológica y no una regla de competencia.

4 E. Gaillard \& J. Savage, Effects of the Arbitration Agreement, Part 2: Chapter III, en Fouchard Gaillard Goldman on International Commercial Arbitration, 381-416. (E. Gaillard \& J. Savage Eds., Kluwer Law International, Montreal, 1999).

5 La Ley Modelo de CNUDMI enuncia el principio de separabilidad en el Artículo 16(1): "Una cláusula compromisoria que forme parte de un contrato se considerará como un acuerdo independiente de las demás estipulaciones del contrato. La decisión del tribunal arbitral de que el contrato es nulo no entrabará ipso jure la nulidad de la cláusula compromisoria". 
tienen la facultad de resolver controversias relativas a la existencia y validez del pacto arbitral ${ }^{6}$.

Ahora bien, la doctrina $\operatorname{arbitral}^{7}$ ha establecido que el principio de competence-competence tiene una función dual, o un doble efecto si se quiere, sobre el acuerdo arbitral. Por un lado, un efecto positivo consistente en la consagración y el reconocimiento del poder, a favor de los árbitros, de dirimir toda controversia relativa a su propia competencia, esto es, toda controversia en cuanto a la existencia, validez y alcance del convenio arbitral. Por el otro lado, tiene un efecto negativo, el cual determina que los árbitros son los primeros jueces de su competencia $\mathrm{y}$, en consecuencia, las cortes o jueces nacionales deben remitir a arbitraje aquellas controversias derivadas de contratos que incluyan pactos o acuerdos arbitrales.

El efecto positivo tiene plena aceptación en la mayoría de los sistemas legales y ha sido reconocido por diversas legislaciones, convenciones y reglas en materia de arbitraje internacional, tales como la Ley Modelo de Arbitraje de la $\mathrm{CNUDMI}^{8}$, el Convenio sobre Arreglo de Diferencias Relativas a Inversiones entre Estados y Nacionales de Otros Estados (CIADI) ${ }^{9}$, las Reglas de Arbitraje

6 E. Silva Romero, Breves observaciones sobre el principio de Kompetenz-Kompetenz en El contrato de arbitraje, 579-668 (E. Silva \& F. Mantilla, Eds., Legis, Bogotá D. C., 2008).

7 A este respecto, Gaillard, Óp. cit., p. 400.

8 La lMa (Nueva York, 2008) consagra el principio de competence-competence en su aspecto positivo en el Artículo 16 numerales 1 y 3. El Artículo 16(1) señala: "El tribunal arbitral estará facultado para decidir acerca de su propia competencia, incluso sobre las excepciones relativas a la existencia o a la validez del acuerdo de arbitraje", y el Artículo 16(3) establece la revisión de la decisión del tribunal arbitral por las cortes nacionales, y determina que si " $\mathrm{el}$ tribunal arbitral se declara competente, cualquiera de las partes, dentro de los treinta dias siguientes al recibo de la notificación de esa decisión, podrá solicitar del tribunal competente conforme al artículo 6 que resuelva la cuestión, y la resolución de este tribunal será inapelable; mientras esté pendiente dicha solicitud, el tribunal arbitral podrá proseguir sus actuaciones $y$ dictar un laudo". La nota explicativa de la Ley Modelo, en su revisión de 2006, determina precisamente la adopción en el Artículo 16(1) del principio de competence-competence y la supervisión judicial que sigue a la decisión adoptada por el tribunal arbitral sobre su propia competencia.

9 Centro Internacional de Arreglo de Diferencias Relativas a Inversiones (Washington, 2006) El convenio CIADI, se refiere al principio de competence-competence de manera expresa en el Artículo 41, el cual establece: “(1) El Tribunal resolverá sobre su propia competencia. (2) Toda alegación de una parte que la diferencia cae fuera de los límites de la jurisdicción del Centro, o que por otras razones el Tribunal no es competente para oírla, se considerará por el Tribunal, el que determinará si ha de resolverla como cuestión previa o conjuntamente con el fondo de la cuestión". 
de la $\mathrm{CCI}^{10}$, las Reglas de Arbitraje del Centro Internacional para la Resolución de Disputas (CIRD) ${ }^{11}$ y el Reglamento de Arbitraje de la CNUDMI ${ }^{12}$.

Por su parte, el efecto negativo del principio de competencecompetence también es aceptado y reconocido por diversas convenciones internacionales, en especial, en el Artículo 8(1) de la Ley Modelo y Artículo II (3) de la Convención de Nueva York de $1958^{13}$. Vale la pena anotar que este efecto negativo genera mayores controversias a la hora de determinar el alcance de la revisión que las cortes pueden realizar sobre la existencia y validez del acuerdo arbitral, tópico al cual volveremos más adelante.

10 Cámara de Comercio Internacional. Las reglas de arbitraje de la CCI (París, 2011) hacen alusión al principio de competence-competence, en el Artículo 6(2), donde señala que toda cuestión de jurisdicción será decidida directamente por el tribunal arbitral. Dentro de las reglas de arbitraje de la CCI, sí existe controversia sobre la existencia y validez del acuerdo arbitral planteada por alguna de las partes; el Secretario General puede remitir el asunto a la Corte de la CCI para que esta decida prima facie la posible existencia del acuerdo arbitral.

11 En las reglas de arbitraje del CIRD (Nueva York, 2010) el principio de competence-competence se ve reflejado en el Artículo 15, numeral 1, que señala: "El tribunal está facultado para decidir sobre su propia jurisdicción, incluyendo cualesquiera objeciones con respecto a la existencia, alcance o validez del acuerdo de arbitraje".

12 Las reglas de arbitraje de la CNUDMI (Nueva York, 2011) versión revisada 2010, establecen el principio en mención en el Artículo 23, numeral 1, al referirse a la declinatoria de la competencia del tribunal arbitral: "El tribunal arbitral estará facultado para decidir acerca de su propia competencia, así como acerca de toda excepción relativa a la existencia o a la validez de un acuerdo de arbitraje. A ese efecto, una cláusula compromisoria que forme parte de un contrato se considerará un acuerdo independiente de las demás estipulaciones del contrato. La decisión del tribunal arbitral de que el contrato es nulo no entrañará ipso jure la nulidad de la cláusula compromisoria".

13 El Artículo 8(1) establece: “1) El tribunal al que se someta un litigio sobre un asunto que es objeto de un acuerdo de arbitraje remitirá a las partes al arbitraje si lo solicita cualquiera de ellas, a más tardar, en el momento de presentar el primer escrito sobre el fondo del litigio, a menos que se compruebe que dicho acuerdo es nulo, ineficaz o de ejecución imposible. 2) Si se ha entablado la acción a que se refiere el párrafo 1) del presente artículo, se podrá, no obstante, iniciar o proseguir las actuaciones arbitrales y dictar un laudo mientras la cuestión esté pendiente ante el tribunal". La nota explicativa de la Ley Modelo, señala que el Artículo 8(1) sigue el modelo del Artículo II (3) de la Convención de Nueva York de 1958, al señalar que el tribunal judicial deberá remitir "a las partes al arbitraje si se le presenta una reclamación sobre un asunto que sea objeto de un acuerdo de arbitraje, a menos que se compruebe que el acuerdo de arbitraje es nulo, ineficaz o de ejecución imposible". 


\section{RELACIÓN DEL PRINCIPIO DE COMPETENCE- COMPETENCE Y LAS TÁCTICAS DILATORIAS}

La relación del principio de competence-competence con las tácticas dilatorias empleadas por las partes en el proceso arbitral, la hallamos en el análisis de las finalidades o de las motivaciones que han llevado a su implementación en la doctrina arbitral. De acuerdo con Silva" ${ }^{14}$, el objetivo que encarna este principio "es proteger la autonomía y la independencia de la justicia arbitral con relación a la justicia estatal, y por esa vía, lograr que la justicia arbitral sea verdaderamente eficaz".

En ese orden de ideas, este principio busca evitar la implementación de tácticas dilatorias del procedimiento arbitral por alguna de las partes, de tal manera que "el poder del árbitro para determinar su propia jurisdicción está justificado por el deseo de facilitar la debida administración de justicia (...) Así se evita retrasar los procedimientos mientras se espera por una decisión con respecto a la jurisdicción, atiborrar los tribunales estatales y prevenir tácticas dilatorias"15.

Al respecto, la nota explicativa de la Ley Modelo hace alusión a esta finalidad, al indicar que cuando el tribunal arbitral decide sobre su propia competencia como cuestión previa, "el Art. 16 nos plantea tres formas de reducir el riesgo y los efectos de las tácticas dilatorias: (1) un plazo breve para recurrir al tribunal judicial (30 días), (2) la inapelabilidad de la resolución del tribunal judicial y, (3) la facultad discrecional del tribunal arbitral de proseguir las actuaciones y dictar un laudo mientras esté pendiente la cuestión ante el tribunal judicial"'. (Resaltado fuera del original)

De igual forma, la relación entre el principio de competencecompetence y las tácticas dilatorias también puede ser determinada según sus efectos positivos como negativos

14 E. Silva Romero, Breves observaciones sobre el principio de Kompetenz-Kompetenz en El contrato de arbitraje, 579 (E. Silva \& F. Mantilla. Eds., Legis, Bogotá D.C., 2008).

15 M. Philippe, The Powers of the Arbitrator and of the ICC Court of Arbitration in Relation to their Jurisdiction. En http://www.arbitralwomen.org/files/publication/23091511521052.pdf (2006).

16 Ley Modelo de Arbitraje de la CNUDMI. 
A. Efecto positivo del principio de competencecompetence y su relación con las tácticas dilatorias

Desde el punto de vista del efecto positivo del principio, los árbitros están empoderados para regular su propia jurisdicción, y ese poder del tribunal arbitral de examinar su propia competencia evita que las partes - al interponer recursos ante cortes nacionales con el alegato de la falta de jurisdicción del tribunal o la nulidad del contrato principal o del acuerdo arbitral- demoren la constitución del tribunal o el avance del procedimiento arbitral. Al respecto, por ejemplo, las reglas de arbitraje de la CCI previenen el uso de tácticas dilatorias al deferir el examen de la cláusula arbitral a la corte de la CCI. Así, si una de las partes se rehúsa a participar en los procedimientos, impugna la jurisdicción de los árbitros (con o sin fundamento que lo justifique) o emplea cualquier otro tipo de tácticas dilatorias, una vez la corte de la CCI decide darle vía libre al arbitramento, el propio tribunal arbitral decidirá su propia competencia sin la injerencia de las cortes nacionales ${ }^{17}$.

A su vez, las reglas de arbitraje de la CIRD, al consagrar el principio de competence-competence, previenen el empleo de tácticas dilatorias al limitar la posibilidad de objeciones a la jurisdicción del tribunal o a la arbitrabilidad del fondo de la controversia, al establecer que sean expuestas a más tardar en el escrito de contestación de la demanda o en la demanda de reconvención ${ }^{18}$. Similar solución prevén las reglas de arbitraje de la CNUDMI, en el numeral 2, del Artículo 23: "La excepción de incompetencia del tribunal arbitral deberá oponerse a más tardar en el momento de presentar la contestación o, con respecto a una reconvención o a una demanda a efectos de compensación, en la réplica a esa reconvención o a la demanda a efectos de compensación". Este artículo, también agrega otra forma de prevenir las tácticas dilatorias de

17 M. Philippe, The Powers of the Arbitrator and of the ICC Court of Arbitration in Relation to their Jurisdiction. En http://www.arbitralwomen.org/files/publication/23091511521052.pdf (2006).

18 Artículo 15.3 de las Reglas de Arbitraje de la CIRD. 
las partes, al expresar que "la excepción basada en que el tribunal arbitral ha excedido su mandato deberá oponerse tan pronto como se plantee durante las actuaciones arbitrales la materia que supuestamente exceda su mandato. El tribunal arbitral podrá, en cualquiera de los casos, estimar una excepción presentada más tarde si considera justificada la demora".

B. Efecto negativo del principio de competence competence y su relación con las tácticas dilatorias

Ahora bien, con respecto al efecto negativo del principio de competence-competence, la doctrina ${ }^{19}$ ha señalado que "el efecto negativo puede ser considerado como parte de un arsenal de herramientas doctrinales para combatir las tácticas de una parte que desea sabotear los procedimientos". Así las cosas, cuando las cortes, en virtud del principio de competence-competence, declinan su jurisdicción en razón de la existencia de un acuerdo arbitral, esto permite que las partes que quieran retrasar en forma injustificada un proceso arbitral no lograrán su cometido, en la medida en que el juez o la corte deban rechazar cualquier acción tendiente a impugnar la jurisdicción del tribunal, o cuestionar la validez del acuerdo arbitral o del contrato principal.

Podemos señalar como ejemplo, el Artículo 23 numeral 4, de las reglas de arbitraje de la Corte de Arbitraje Internacional de Londres (LCIA, por sus siglas en inglés) ${ }^{20}$, que consagra el efecto negativo del principio de competence- competence y con esto nos provee de una herramienta estratégica para combatir tácticas dilatorias dentro del proceso arbitral.

El mencionado artículo nos plantea que cuando las partes aceptan arbitrar bajo las reglas de la LCIA, se entiende que han

19 E. Gaillard en W. Park, The Arbitrator's Jurisdiction to Determine Jurisdiction in International Arbitration 2006: Back to Basics? 55-153 (A. J. van Den Berg Ed., Kluwer Law International, Montreal, 2006).

20 LCIA (1998). El Artículo 23 numeral 4 dice: "By agreeing to arbitration under these rules, the parties shall be treated as having agreed not to apply to any state court or other judicial authority for any relief regarding the Arbitral Tribunal's jurisdiction or authority, except with the agreement in writing of all parties to the arbitration or the prior authorization of the Arbitral Tribunal or following the latter's award ruling on the objection to its jurisdiction or authority". 
acordado de forma contractual no acudir a cortes estatales o utilizar recursos judiciales de ninguna índole para discutir la jurisdicción o autoridad del tribunal arbitral o contra el subsiguiente laudo, lo que regula la objeción a su jurisdicción o autoridad. En este evento, si una de las partes presenta una acción de este tipo ante una corte o tribunal judicial, incurriría en incumplimiento del contrato con el subsecuente reclamo por daños basado en los costos incurridos por la prematura acción ante la Corte ${ }^{21}$.

La importancia del efecto negativo del principio de competence-competence radica entonces, en evitar la interrupción del proceso arbitral por la sola afirmación de una de las partes sobre la inexistencia o nulidad del pacto arbitral, y la intervención de la justicia estatal antes que los árbitros alcancen una decisión sobre su propia competencia y resuelvan sobre los méritos de la controversia.

Ahora bien, antes había sido mencionado que sobre el efecto negativo del principio de competence-competence existe una discusión, planteada en la doctrina arbitral, sobre el alcance de la revisión por parte de las cortes judiciales sobre la decisión del tribunal arbitral. La observación consiste en discernir si la revisión judicial debe hacerse prima facie o en forma total, es decir, abordar no solo el asunto de la jurisdicción del tribunal arbitral sino también la validez o existencia del acuerdo arbitral.

Este análisis es importante porque si se considera que el alcance de la revisión judicial debe ser total, se lograría definir con certeza la jurisdicción del tribunal arbitral; esto representaría para las partes un ahorro en tiempo y costos, mientras que el tribunal arbitral y las cortes no se desgastarían en llevar un proceso cuya decisión, a la larga, podría ser impugnada, anulada o imposible de ejecutar. Por otro lado, incentivaría el uso de tácticas dilatorias por las partes renuentes al avance del proceso arbitral.

21 J. Morrissey \& J. Graves, International Sales Law and Arbitration. Problems, Cases and Commentary, 383 (Aspen Publishers, Frederick, Maryland, 2008). 
Ahora, si se considera que el alcance de la revisión judicial debe ser prima facie, entonces el juez o la corte limitarían su revisión a determinar de manera preliminar si la objeción presentada tiene fundamento o no. En este caso, se aseguraría que las partes que buscan obstruir el arbitraje no estén habilitadas para ello ni alegar ante las cortes procedimientos relativos a la existencia, alcance y validez del acuerdo arbitral. Aunque, por otro lado, no brinda certeza jurídica sobre la decisión tomada por el tribunal, lo que puede causar más costos y demoras.

La Corte Suprema de Justicia de Canadá, se refirió a esta discusión en el caso Dell Computer Corp. vs. Union des consommateurs $^{22}$, que planteó una controversia sobre si los derechos del consumidor eran arbitrables o no bajo las leyes de la provincia de Quebec. En su razonamiento, la Corte expuso el significado y los argumentos a favor y en contra de la revisión judicial basada en el "enfoque prima facie", y el "enfoque comprehensivo", con base en los argumentos presentados por ambas partes dentro del proceso $^{23}$.

Con respecto al enfoque prima facie, la Corte concluyó que este debe interpretarse en el siguiente sentido: la corte que recibe la impugnación debe decidir si el acuerdo arbitral aparenta ser válido y aplicable a la disputa, solo sobre la base de los documentos producidos para apoyar la acción, y presumir que estos son verdaderos sin escuchar ninguna evidencia testimonial. En adición, la Corte señaló que la decisión de las cortes en este tipo de impugnaciones jurisdiccionales no tiene la autoridad de una sentencia final, por tanto, el tribunal arbitral puede conducir su propia revisión comprehensiva de la validez del arbitramento,

22 Dell Computer Corp. vs. Union des consommateurs. 2 SCR 801, SCC 34 (2007).

23 Con respecto al enfoque que finalmente se acoge en Canadá, el autor Gary Born explica que la tendencia de las cortes canadienses no es unánime y al parecer cada una tiene discreción de escoger el enfoque que le parezca conveniente según el tipo de problema jurídico. Con respecto a la decisión del caso Dell, la regla que la Corte determinó es que debe aplicarse el enfoque prima facie si los problemas jurídicos se relacionan con cuestiones de hecho, o con cuestiones de hecho y derecho, y el enfoque comprehensivo si los problemas jurídicos se relacionan con cuestiones de derecho. G. Born, International Arbitration Agreements and Competence-Competence. Variations of Competence-Competence. Doctrine in Different National Legal Regimes, en International Commercial Arbitration, 877-986 (Kluwer Law International, Alphen aan den Rijn, 2009). 
sujeta a la revisión subsecuente de las cortes. En relación con el enfoque comprehensivo, la Corte señaló:

"The advocates of a comprehensive judicial review of the validity of the arbitration agreement (...) rely on an 'economy-of-means' rationale. They argue that it is a waste of time and money to refer the question of the validity of an agreement to an arbitral tribunal, whose very jurisdiction is challenged by one of the parties, in order to allow it to first rule on the question, as the parties will always invariably have to return to the court either for a decision in the validity of the arbitration agreement (...) (if the arbitral tribunal has declared itself competent) or to continue the proceedings that were interrupted by the referral application (if the arbitral tribunal has declared itself incompetent). They also argue that, as the jurisdiction of the arbitral tribunal depends entirely on the validity of the arbitration, it is illogical to ask the arbitral tribunal to first rule on the validity of the arbitration agreement" ${ }^{\prime 24}$.

De igual forma, la Corte resaltó que un argumento principal a favor del enfoque prima facie es la prevención de las tácticas dilatorias, puesto que una revisión comprehensiva de la validez de un acuerdo arbitral, basada en evidencia testimonial y documental, puede tomar meses para llegar a una decisión, y permitir tal revisión le daría a una parte recalcitrante la oportunidad de demorar de forma indebida el inicio o el progreso del arbitraje. Es más la Corte, en el párrafo 86 de la sentencia, aclaró que esa corporación en primera instancia debe estar satisfecha con encontrar que la impugnación a la jurisdicción del tribunal arbitral no es una táctica dilatoria, ni impedirá el desarrollo del procedimiento arbitral.

Este punto de vista, encuentra apoyo en otros autores como Frédéric Bachand ${ }^{25}$, quien plantea que la política detrás del enfoque prima facie es darle prioridad a la prevención de tácticas dilatorias, en orden de asegurar la eficiencia del arbitraje comercial internacional. Esta posición se justifica por dos razones principales: una, la revisión de una corte de la jurisdicción del

24 Sentencia 34, pár. 170 (2007).

25 El autor cita a Emmanuel Gaillard entre aquellos que apoyan la aplicación del enfoque prima facie. F. Bachand, Does Article 8 of the Model Law Call for Full or Prima Facie Review of the Arbitral Tribunal's Jurisdiction? 22 Arbitration International, 3, 465 (Kluwer Law International, 2006). 
tribunal arbitral puede prolongarse demasiado tiempo, lo que se suma a los recursos de apelación que puedan estar disponibles; $\mathrm{y}$ dos, permitir una revisión comprehensiva de la jurisdicción del tribunal arbitral, le da oportunidad a aquella parte que no quiere arbitrar para retardar el proceso arbitral mediante apelación a la intervención judicial ${ }^{26}$.

Vale la pena acotar que, en todo caso, el enfoque comprehensivo ha sido acogido por cortes y jueces en varias jurisdicciones, como Canadá, Hong Kong, Nueva Zelanda y Australia, donde se ha planteado que debe hacerse una revisión total sobre la validez y aplicabilidad del acuerdo arbitral, cuando conocen de recursos o acciones fundamentadas en argumentos de falta de jurisdicción del tribunal arbitral ${ }^{27}$.

Sin embargo, a pesar de la divergencia que existe en la interpretación de los artículos 8(1) de la Ley Modelo y II(3) de la Convención de Nueva York de 1958 — que permiten a las cortes revisar los méritos de una disputa sin referir a las partes a un arbitraje - si encuentran que el acuerdo arbitral es "nulo, ineficaz o de ejecución imposible", hay una tendencia creciente a considerar que la revisión de las cortes sobre la jurisdicción del tribunal arbitral debe ser prima facie, con el fundamento de evitar la práctica de tácticas dilatorias en el proceso arbitral $^{28}$. En relación con este tema, Emmanuel Gaillard ${ }^{29}$ ha señalado que es más convincente seguir el enfoque prima facie, precisamente por las consideraciones de política detrás del efecto negativo del principio de competence-competence: la prevención de tácticas dilatorias y la centralización del litigio relativo a la existencia y validez del acuerdo arbitral ante ciertas cortes.

\footnotetext{
26 F. Bachand, Óp. cit., p. 465.

27 Ibídem, p. 463.

$28 \mathrm{Al}$ respecto, Frédéric Bachand, señala "que como la relación entre las partes envueltas en un procedimiento arbitral internacional tiende a ser hostil, una política que restringa, en las primeras etapas del procedimiento, la intervención judicial en objeciones jurisdiccionales en orden a limitar la oportunidad de que tales tácticas dilatorias, es cada vez más atractiva". Ibid.

29 Gaillard, Óp. cit., pp. 411-412.
} 


\section{Tácticas dilatorias relacionadas con la impugnación a la competencia del tribunal arbitral}

La alegación ante cortes nacionales sobre la falta de autoridad del tribunal arbitral para conocer de la controversia presentada ante este, es una de las tácticas dilatorias más comunes empleadas dentro del arbitraje comercial internacional. Al respecto, la parte que impugna la competencia del tribunal por lo general utiliza como fundamento de su alegación el supuesto incumplimiento de alguno de los requisitos necesarios para la existencia o validez del acuerdo arbitral.

De esta forma, las alegaciones pueden ser muy variadas, por ejemplo: falta de firma del pacto arbitral por una de las partes; la falta de identidad entre quien firmó el pacto arbitral y quien hace parte del proceso; la ineficacia o inaplicabilidad del pacto arbitral; el desbordamiento de la competencia del tribunal arbitral al aplicar el pacto arbitral; la falta de constancia por escrito del pacto arbitral; la inarbitrabilidad de la disputa; o la incapacidad de una de las partes.

Sin desconocer que la falta de competencia del tribunal arbitral es un argumento que puede ser utilizado de buena fe y en forma justificada, no es menos cierto que en muchos casos el móvil real lo constituye la intención deliberada de las partes de incumplir el acuerdo arbitral. Al respecto, Fernando EstavilloCastro señala que en muchas ocasiones, a pesar de existir un acuerdo arbitral incuestionable, válido y ejecutable, una de las partes decide iniciar una acción judicial, con conciencia absoluta no solo de la demora que esto ocasionará al proceso sino también de los costos en que deberá incurrir la contraparte para lidiar con la demanda paralela (parallel suit); el autor resalta que este tipo de actitud puede ser equivalente a la "denegación de la justicia"30.

Un ejemplo de las maniobras obstructivas que pueden ser empleadas en el arbitraje institucional, es la insistencia de una de

30 F. Estavillo-Castro, Ethics in Arbitration en Liber Amicorum Bernardo Cremades, 387-411 (M. Á. Fernández-Ballesteros \& David Arias, Eds., La Ley, Madrid, 2010). 
las partes de acudir a instancias judiciales aunque la institución arbitral determine en sus reglas el procedimiento para cuestionar la existencia del pacto arbitral; esto, incluso cuando ya se ha agotado el recurso ante la respectiva institución de arbitraje, o cuando este todavía se encuentra en curso. Está táctica puede combatirse tal como lo señala el Artículo 6(2) del reglamento de arbitraje de la $\mathrm{CCI}^{31}$, el cual establece que estas acciones serán puestas, en primera instancia, en conocimiento de la corte de la CCI para que sea esta la que examine la existencia, validez o alcance del convenio arbitral. Si la Corte encuentra que el acuerdo es inexistente entonces las partes se encontrarán en libertad de acudir a la jurisdicción competente.

Por otro lado, es común encontrar partes que solo participan en el proceso arbitral para alegar la falta de competencia del tribunal arbitral, con el fin de retardar los procedimientos. En la actualidad, esta actitud se combate con el otorgamiento a los árbitros de la facultad de continuar con el proceso arbitral, sin la participación de la parte renuente ${ }^{32}$. Así sucedió, en el caso Texas Overseas Petroleum Co. y Californian Asiatic Oil Company vs. República Árabe de Libia: al recibir la notificación de la solicitud de arbitraje, el gobierno de Libia la rechazó y no nombró al árbitro que le correspondía de acuerdo con lo pactado en el contrato. Por tal motivo, los demandantes, con fundamento en una de las provisiones del contrato de concesión, solicitaron el nombramiento de árbitro único al Presidente de la Corte Internacional de Justicia, entidad que procedió al nombramiento ${ }^{33}$.

31 "Cuando una de las partes plantea uno o varias propuestas relativas a la existencia, validez, o alcance del convenio arbitral, la Corte puede decidir, sin prejuzgar la admisibilidad o el fundamento de tales propuestas, que el arbitraje tenga lugar si, prima facie, estima posible la existencia de un convenio arbitral acomodado al reglamento. En este caso, corresponderá al tribunal arbitral decidir sobre su propia competencia. Si la Corte no llega a esta conclusión, las partes son informadas de que el arbitraje no puede tener lugar. En este caso las partes mantienen su derecho de dirigirse ante la jurisdicción competente según que estén o no vinculadas por un convenio arbitral".

32 "It is generally accepted today that the refusal of a party to participate in arbitration proceedings, such party's strategic's default, is of no use if arbitrators decide disputes without hearing the points of view of the defaulting party". En F. Estavillo-Castro, Óp. cit., pp. 387-411.

33 Sentencia arbitral de 12 de enero de 1977, asunto Texaco-Calasiatic contra gobierno de Libia, en M. Janis \& J. Noyes, International Law. Cases and Commentary, 117-126 (3 ${ }^{\mathrm{a}}$. ed., Thompson West, St. Paul, 2006). 
De igual forma, también es posible encontrar que las partes esperan hasta que el laudo arbitral sea emitido para interponer el recurso de anulación en su contra, con el fundamento de la falta de competencia del tribunal arbitral. En muchas ocasiones, esta actitud se trata de una simple táctica o estrategia para obstruir el reconocimiento y ejecución de laudo. Por tal motivo, algunas legislaciones, como la inglesa, prevén que si una parte no ha objetado la falta de competencia del tribunal arbitral, no podrá hacerlo más tarde. Así la sección 73 del English Arbitration Act de 1996 señala que una parte en un procedimiento arbitral puede perder su derecho a objetar la falta de competencia del tribunal arbitral ante el tribunal o una corte, si no lo invocado en el tiempo señalado para ello ${ }^{34}$.

Esta misma regla se aplicó en el caso CTA Lind \& Co. Scandinavia AB in Liquidation's Bankruptcy Estate vs. Erik Lind, por la Corte de Distrito de los Estados Unidos para el Distrito Medio de Florida ${ }^{35}$. El demandado, mediante una declaración escrita, manifestó que no tenía objeción a la jurisdicción del tribunal arbitral pero no compareció a la audiencia respectiva. El laudo arbitral fue emitido a favor de la parte demandante y

34 La sección 73 dice: "Loss of right to object. (1) If a party to arbitral proceedings takes part, or continues to take part, in the proceedings without making, either forthwith or within such time as is allowed by the arbitration agreement or the tribunal or by any provision of this Part, any objection:

(a) that the tribunal lacks substantive jurisdiction,

(b) that the proceedings have been improperly conducted,

(c) that there has been a failure to comply with the arbitration agreement or with any provision of this Part, or

(d) that there has been any other irregularity affecting the tribunal or the proceedings, he may not raise that objection later, before the tribunal or the court, unless he shows that, at the time he took part or continued to take part in the proceedings, he did not know and could not with reasonable diligence have discovered the grounds for the objection.

(2) Where the arbitral tribunal rules that it has substantive jurisdiction and a party to arbitral proceedings who could have questioned that ruling:

(a) by any available arbitral process of appeal or review, or

(b) by challenging the award,

does not do so, or does not do so within the time allowed by the arbitration agreement or any provision of this Part, he may not object later to the tribunal's substantive jurisdiction on any ground which was the subject of that ruling".

35 United States District Court, Middle District of Florida, Tampa Division, CTA Lind \& Co. Scandinavia AB in Liquidation's Bankruptcy Estate vs. Erik Lind, No. 665, case 8:08-cv-1380-T30TGW (April 7, 2009). XXXIV Yearbook Commercial Arbitration (Kluwer Law International, The Hague, 2009). 
esta buscó la respectiva ejecución. El demandado se opuso a la confirmación del laudo, con el argumento de la inexistencia de un acuerdo para arbitrar, en específico, porque no firmó el contrato en su capacidad individual. La Corte distinguió este caso del caso Czarina ${ }^{36}$, y señaló que el demandado renunció a cualquier objeción a la jurisdicción del tribunal, al haberlo declarado por escrito.

Esta misma Corte también sostuvo que cuando la parte que busca la confirmación cumple con su carga de jurisdicción se establece un caso prima facie para la confirmación del laudo, y se invierte la carga de la prueba de modo que el demandado debe establecer la invalidez del laudo con fundamento en alguna de las causales especificadas en el Artículo V de la Convención de Nueva York.

Como respuesta a la utilización abusiva de tácticas dilatorias, algunas cortes han señalado que es posible castigar a las partes mediante la condena al pago de los costos en que la otra parte ha incurrido para defenderse de esas tácticas. Así, en Estados Unidos, aunque solo en extremas circunstancias, las cortes han establecido la posibilidad de sancionar económicamente a las partes que actúan de mala fe en el proceso arbitral.

En el caso Trans Chemical Limited vs. China National Machinery Import and Export Corporation, ante la Corte de Distrito de los Estados Unidos para el Distrito Sur de Texas ${ }^{37} 34$, esta instancia sostuvo que - aunque el Federal Arbitration Act no otorga honorarios de abogados a la parte que vence en su acción para la confirmación de un laudo arbitral en una corte federal - la parte vencedora puede tener derecho a honorarios.

36 United States Court of Appeals. 11 ${ }^{\text {th }}$ Circuit. Czarina, LLC vs. W. F. Poe Syndicate, 358 F.3d 1286, 1291. 2004. En este caso, la Corte estudió la solicitud de confirmación de un laudo en que el acuerdo arbitral no constaba por escrito. La parte que buscaba la confirmación del laudo se apoyó en dos casos de confirmación de laudos en los que omitió el mencionado requisito del acuerdo por escrito, porque las partes actuaron en el arbitramento sin impugnar la jurisdicción del tribunal. La corte rechazó este argumento porque la parte que se oponía a la confirmación había objetado a tiempo y frecuentemente el arbitraje y nunca había acordado arbitrar la disputa.

3734 United States District Court, Southern District of Texas, Houston Division, Trans Chemical Limited vs. China National Machinery Import and Export Corporation, No., 97-20695. $5^{\text {th }}$ Circuit (July 7, 1997). XXIII Yearbook Commercial Arbitration (Kluwer Law International, The Hague. 1998). 
Esto, si los argumentos expuestos por la parte opositora para impugnar el laudo, carecen de mérito o justificación, o son legalmente frívolos, esto es, presentadas de mala fe para hostigar en vez de ganar. La Corte, en todo caso, enfatizó en el carácter extraordinario de la medida para no desestimular el uso del arbitraje internacional.

Este misma regla fue resaltada en Toyo Tire Holdings of Americas, Inc. vs. Continental Tire North America, Inc. AS ${ }^{38}$ : la Corte al afirmar que las partes en el arbitramento tienen usualmente el incentivo de demorar los procedimientos arbitrales para su propio beneficio, - $-\mathrm{y}$ citó el caso Int'1 Union of Petroleum $\&$ Indus. Workers vs. W. Indus. Maint., Inc. ${ }^{39}$ - señaló que es posible imponer honorarios de abogados contra la parte que se rehúsa a cumplir el laudo arbitral, con el fin de detener tácticas dilatorias frívolas que no solo niegan el derecho a una pronta reparación individual sino que también amenazan el objetivo de la paz industrial"'40.

Otros casos de cortes en Estados Unidos que abordan sobre el mismo tema son: Prospect Capital Corp. vs. Enmon; $c f$. Manning vs. Smith, Barney, Harris Upham \& Co., y Quick \& Reilly, Inc. vs. Jacobson, casos que establecen sanciones por la impugnación sin mérito de laudos arbitrales ${ }^{41}$.

En otra instancia, en India, la Alta Corte de Bombay, en el caso Renusagar Power Company vs. General Electric Company, al confirmar la ejecución de un laudo arbitral de la CCI.

38 United States Court of Appeals. $9^{\text {th }}$ Circuit, Toyo Tire Holdings of Americas, Inc. vs Continental Tire North America, Inc., WL 2496133 (June 17, 2010).

39 United States Court of Appeals. $9^{\text {th }}$ Circuit, Int'l Union of Petroleum \& Indus. Workers vs. W. Indus. Maint., Inc., 707 F.2d 425, 428 (1983).

40 Texto original: "Moreover, one party to the arbitration often has an incentive to delay arbitration proceedings to its own advantage. See, e.g., Int'l Union of Petroleum \& Indus. Workers v. W. Indus. Maint., Inc., 707 F.2d 425, 428 (9th Cir. 1983) (upholding award of attorney's fees against party who refused to abide by arbitrator's award to deter " frivolous dilatory tactics [which] not only den[y] the individual promptredress, [but also] threaten[...] the goal of industrial peace)".

41 SDNY, Prospect Capital Corp. vs. Enmon, No. 08-cv-03721, 2010 BL 185181, at *14-15 (Mar. 9, 2010); SDNY, $c f$. Manning vs. Smith, Barney, Harris Upham \& Co., 822 F. Supp. 1081, 1083-84 (June 9, 1993); y SDNY, Quick \& Reilly, Inc. vs. Jacobson, 126 FRD 24, 27 (May 25, 1989), en S. Koh \& J. Hager, Ordering Cost to Temper the "Americanization" of International Commercial Arbitration en Bloomberg Law Reports, 2010. En http://www.perkinscoie.com/files/upload/ COMM_10_08_OrderingCostsArticle.pdf (August 8, 2010). 
La instancia señaló que no puede considerarse una decisión como esta contraria al orden público, cuando concede a la parte vencedora grandes costos en los que ha incurrido durante el proceso arbitral, si esos costos se deben a las tácticas dilatorias y pequeñas objeciones técnicas adoptadas por la otra parte, es decir, que estas generaron la prolongación del proceso arbitral y el incremento de sus $\operatorname{costos}^{42}$.

En referencia a esta última afirmación, se debe mencionar que instituciones arbitrales como la LCIA, la CCI y la CIRD mantienen provisiones que autorizan al tribunal arbitral a determinar la proporción de los costos que cada una de las partes debe solventar.

Así, el Artículo 28(3) de las reglas de arbitraje de la LCIA dispone que el Tribunal Arbitral tiene el poder de imponer costos a una de las partes, total o parcialmente, legales o de otro tipo, en que haya incurrido la otra parte. El Artículo 31(3) de las reglas de arbitraje de la CCi consagra que en laudo final se fijarán los costos del arbitraje y se decidirá cuál de las partes debe pagarlos o en qué proporción deben repartirse. El Artículo 31 de las reglas de arbitraje internacional de la CIRD, señala: "El tribunal fijará las costas del arbitraje en su laudo. El tribunal podrá imponer todas o parte de las costas a una de las partes si considera que esa imposición es razonable, tomando en cuenta las circunstancias del caso".

Es posible concluir entonces, que la utilización de tácticas dilatorias para frenar el avance del procedimiento arbitral o para demorar el reconocimiento y ejecución de un laudo arbitral, puede ser manejada por las cortes bien sea por medio de la imposición de costos de litigio a las partes infractoras, o mediante la confirmación de los costos con que los tribunales arbitrales las hayan gravado.

42 High Court, Bombay, Renusagar Power Company vs. General Electric Company, No. 19 (October 12, 1989). XVI Yearbook Commercial Arbitration (Kluwer Law International, The Hague, 1991). 


\section{CONCLUSIÓN}

Es claro que el empleo de tácticas dilatorias dentro del proceso arbitral, y en especial aquellas dirigidas a cuestionar la competencia del tribunal arbitral, se ha convertido en uno de los principales problemas en el desarrollo del arbitraje comercial internacional. Combatir dichas prácticas es uno de los mayores retos de quienes participan en este pequeño pero agitado foro, en particular, para quienes fungen como árbitros o abogados de partes y para aquellas cortes o tribunales judiciales que reciben acciones interpuestas por partes recalcitrantes.

El principio de competence-competence, como pieza fundamental del arbitraje comercial internacional, tanto desde su efecto positivo como negativo, se alza como la solución más efectiva para prevenir el uso de estas tácticas dilatorias. El efecto positivo dota al tribunal arbitral con el poder de decidir sobre su propia competencia, y previene que las partes entorpezcan la continuidad del proceso arbitral al interponer recursos ante cortes nacionales. El efecto negativo, al declinar las cortes nacionales su jurisdicción en razón de la existencia de un acuerdo arbitral, causa que los recursos judiciales interpuestos como maniobra obstructiva no tengan éxito.

Así las cosas, tácticas relativas a la impugnación de la competencia del tribunal arbitral sobre la base de la falta de firma del pacto arbitral por una de las partes; la falta de identidad entre quien firmó el pacto arbitral y quien hace parte del proceso; la ineficacia o inaplicabilidad del pacto arbitral; el desbordamiento de la competencia del tribunal arbitral al aplicar el pacto arbitral; la falta de constancia por escrito del pacto arbitral; la inarbitrabilidad de la disputa; y la incapacidad de una de las partes, pueden combatirse mediante disposiciones consagradas en los reglamentos de las diversas instituciones de arbitraje internacional, en convenciones e instrumentos internacionales sobre la materia y, en leyes domésticas de arbitraje internacional. Estas normas, fundadas en el principio de competence-competence, establecen límites temporales para objetar la jurisdicción del 
tribunal, conceden facultades discrecionales al tribunal arbitral para garantizar el normal funcionamiento del proceso arbitral e incluso determinan la posibilidad de imponer sanciones económicas para aquellas partes que abusan de su derecho a accionar.

Esta variedad de soluciones se convierte en un valioso recurso que legisladores, cortes judiciales y tribunales arbitrales deben aprovechar, de tal forma que disminuya la oportunidad de utilizar tácticas dilatorias, y se logre la realización de procesos arbitrales acordes con la voluntad de las partes, beneficiosos para la preservación de la institución del arbitraje y que no generen costos innecesarios debido a la iniciación de procedimientos paralelos ante las cortes nacionales. 


\section{BIBLIOGRAFÍA}

\section{Libros}

Estavillo-Castro, F., Ethics in Arbitration en Liber Amicorum Bernardo Cremades, 387-411 (M. Á. Fernández-Ballesteros \& David Arias, Eds., La Ley, Madrid, 2010).

Janis, M. \& Noyes, J., International Law.Cases and Commentary, 117-126 (3ª ed., Thompson West, St. Paul, 2006).

Morrissey, J. \& Graves, J., International Sales Law and Arbitration. Problems, Cases and Commentary, 383 (Aspen Publishers, Frederick, Maryland, 2008).

Talero Rueda, S., Arbitraje comercial internacional. Instituciones básicas y derecho aplicable, 147 (Ediciones Uniandes, Temis, Bogotá D.C., 2008).

\section{Contribuciones en Obras Colectivas}

Born, G., International Arbitration Agreements and Competence-Competence. Variations of Competence-Competence Doctrine in Different National Legal Regimes, en International Commercial Arbitration, 877-986 (Kluwer Law International, Alphen aan den Rijn, 2009).

Gaillard, E. \& Savage, J., Effects of the Arbitration Agreement, Part 2: Chapter III, en Fouchard Gaillard Goldman on International Commercial Arbitration, 381-416 (E. Gaillard \& J. Savage, Eds., Kluwer Law International, The Hague, 1999).

Fortier, L. Y., The Minimum Requirements of Due Process in Taking Measures Against Dilatory Tactics: Arbitral Discretion In International Commercial Arbitration. A Few Plain Rules And A Few Strong Instincts, en Improving the Efficiency of Arbitration Agreements and Awards: 40 years of Application of the New York Convention, 396-404 (A. J. van Den Berg, Ed., Kluwer Law International, The Hague, 1999).

Park, W., The Arbitrator's Jurisdiction to Determine Jurisdiction en International Arbitration 2006: Back to Basics? 55-153 (A. J. van Den Berg Ed., Kluwer Law International, Montreal, 2006).

Silva Romero, E., Breves observaciones sobre el principio de Kompetenz-Kompetenz en El contrato de arbitraje, 579-668 (E. Silva \& F. Mantilla, Eds., Legis, Bogotá D.C., 2008).

artículos

Bachand, F., Does Article 8 of the Model Law Call for Full or Prima Facie Review of the Arbitral Tribunal's Jurisdiction? 22 Arbitration International, 3, 463-476 (2006). 


\section{Fuentes Digitales}

Koh, S. \& Hager, J., Ordering Cost to Temper the "Americanization" of International Commercial Arbitration. Bloomberg Law Reports, 2010. En http:// www.perkinscoie.com/files/upload/COMM_10_08_OrderingCostsArticle. pdf (August 8, 2010).

Philippe, M., The Powers of the Arbitrator And of the ICC Court Or Arbitration In Relation to their Jurisdiction. En http://www.arbitralwomen.org/files/publication/23091511521052.pdf (2006).

\section{Tratados Internacionales}

Convenio sobre Arreglo de Diferencias Relativas a Inversiones entre Estados y Nacionales de Otros Estados (CIADI). En https://icsid.worldbank.org/ICSID/ StaticFiles/basicdoc-spa/CRR_Spanish-final.pdf (2006).

\section{Leyes e Instrumentos Internacionales}

English Arbitration Act, 1996.

Cámara de Comercio Internacional [CCI]. Reglamento de Arbitraje y ADR, 2012.

Centro Internacional para la Resolución de Disputas [CIRD]. Reglas de Arbitraje, 2010.

Comisión de las Naciones Unidas para el Derecho Mercantil Internacional [CNUDMI]. Ley Modelo sobre Arbitraje Comercial Internacional 1985, con enmiendas aprobadas en 2006.

CNUDMI. Reglamento de Arbitraje, 2010.

Corte de Arbitraje Internacional de Londres. Reglas de Arbitraje, 1998.

Casos

United States Court of Appeals, Czarina, LLC vs. W. F. Poe Syndicate, 358 F.3d 1286, 1291. $11^{\text {th }}$ Cir. 2004.

Canada Supreme Court, Dell Computer Corp. vs. Union des Consommateurs, 2 SCR 801, SCC 34 (2007).

High Court, Bombay, Renusagar Power Company vs. General Electric Company, No. 19 (October 12, 1989). XVI Yearbook Commercial Arbitration (Kluwer Law International, The Hague, 1991).

United States District Court, Fifth Circuit, Southern District of Texas, Houston Division, Trans Chemical Limited vs. China National Machinery Import and Export Corporation, No. 97-20695 (July 7, 1997). XXIII Yearbook Commercial Arbitration (Kluwer Law International, The Hague, 1998). 
United States District Court, Middle District of Florida, Tampa Division, CTA Lind \& Co. Scandinavia AB in Liquidation's Bankruptcy Estate vs. Erik Lind, No. 665, case 8:08-cv-1380-T-30TGW" (April 7, 2009). XXXIV Yearbook Commercial Arbitration (Kluwer Law International, The Hague, 2009).

United States Court of Appeals, Toyo Tire Holdings of Americas, Inc. vs Continental Tire North America, Inc., WL 2496133. $9^{\text {th }}$ Cir. June 17, 2010.

United States Court of Appeals, Int'1 Union of Petroleum \& Indus. Workers vs. W. Indus. Maint., Inc., 707 F.2d 425, 428. $9^{\text {th }}$ Cir. 1983. 